\title{
Research on Surface Fatigue Crack Image Recognition Detection Method Based on Computer Vision Technology
}

\author{
Xuepeng Huang ${ }^{1, a}$, Xiaohui Chen² \\ ${ }^{1}$ Information \& Network Center, Hubei University of Police, P.R. China \\ ${ }^{2}$ Department of Investigation Technology, Hubei University of Police, P.R. China
}

Keywords: Computer vision inspection technology (AVI), Surface fatigue crack, Edge detection, Image recognition.

\begin{abstract}
This paper goes through computer vision technology in the application of image acquisition and processing to solve the surface fatigue crack image recognition detection problem. At the same times, this paper studies machine vision technology (AVI) in the surface fatigue crack detection method, its method goes through crack image to carry on denoising, edge detection, filtering and repair, so as to get more realistic graphics and verify the computer vision technology in the application of surface fatigue crack image recognition detection, the method has achieved the desired results, which makes a breakthrough progress for the surface fatigue crack detection method of computer vision detection technology.
\end{abstract}

\section{Introduction}

With the popularization of computer, the use of computer acquisition and process image information has become a common way, various industrial and agricultural machinery parts wear problems are becoming increasingly prominent in life, the normal use of the machine brings the fatigue crack, which has been buried hidden trouble [1-3]. Therefore, it is very important significance to detect the related part wear and fatigue crack. Based on computer vision technology, this paper designs and explores the mechanical component fatigue crack image recognition detection method, its method has low error, high efficiency, zero contact, real-time monitoring, continuous uninterrupted operation and other advantages [4]. This paper mainly studies the image detection method of mechanical component surface fatigue crack, and the crack image information is obtained by preprocessing the part crack image to determine the length and width of the surface fatigue crack, on the basis of the above, fitting the fatigue crack's length extension characteristics of and width tensile characteristics. For the image preprocessing and crack length calculation, the method also provides a real-time and efficient solution for the detection, identification and prevention analysis of mechanical components surface fatigue cracks.

a Corresponding author : 42482852@qq.com 


\section{Mechanical Components Fatigue Crack Image Identification System Scheme}

For the identification system of the crack image information, the system mainly consists of 7 plates. The paper is first to carry out the image collection and denoising work, and goes through edge detection and threshold segmentation on the image recognition and calculation, and then to modify the computer processing results, so as to extract the required data and make an accurate judgment $[5,6]$. Image information recognition detection system processes are shown in the following diagram.

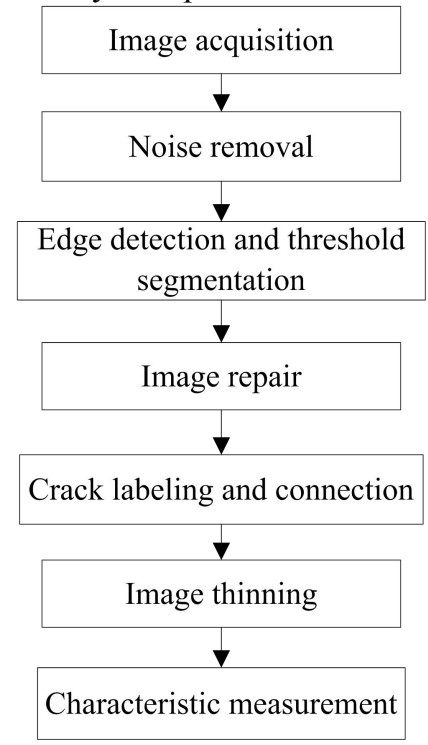

Figure 1. Image information identification detection system flow chart.

\section{The Operation Process of Mechanical Components Fatigue Crack Image Information Identification Detection System}

The image information acquisition mainly goes through the vision sensor, and then the image information is converted to digital information by the image processing system, finally the digital information is saved and processed by the computer processor [7-9]. Surface fatigue crack image information is obtained as shown in Figure 2.

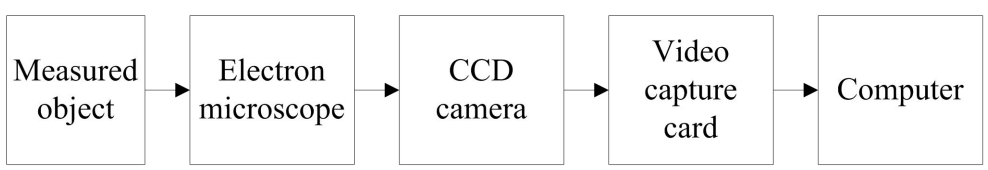

Figure 2. Mechanical surface fatigue crack image information acquisition.

In the actual application, the detected objects are not in the ideal situation, and even some are in very bad environment, we should be noise filtering caused by light, environment and other factors, so that we can better get the image. Comparison before and after image information noise removal is shown in Figure 3. 


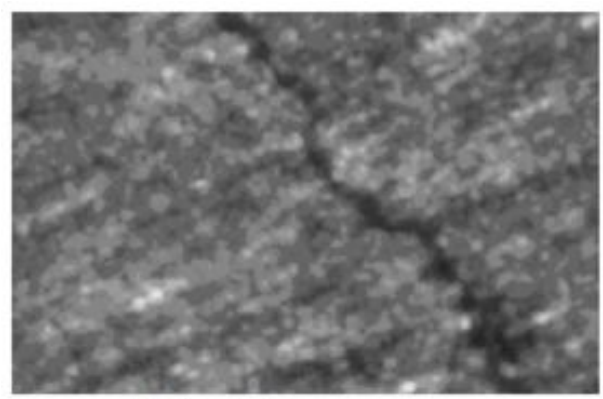

(a) Original image

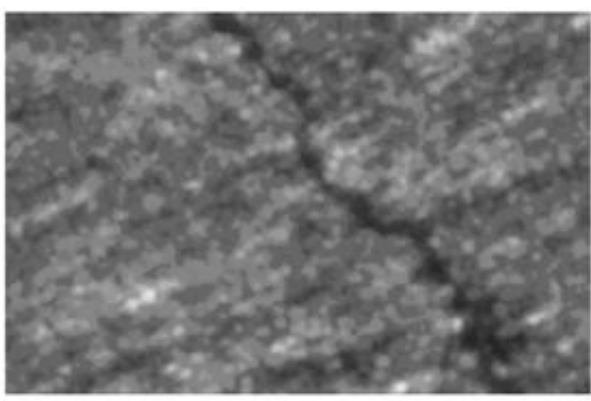

(b) Image after denoising

Figure 3. Comparison chart before and after image information noise removal.

Edge detection and threshold segmentation. Based on the spatial differential operator, the edge detection method will complete the detection template and image information convolution. Because the gray value of the edge is intermittent, the gray value of the image will be changed constantly, and the color will change frequently, the edge crack of the object surface is the detected key link $[10,11]$. In order to detect more accurate, this paper use two kinds of edge crack detection factor, the interaction method using two factors obtain the collection of edge crack points. Edge detection and threshold segmentation process are shown in Figure 4.

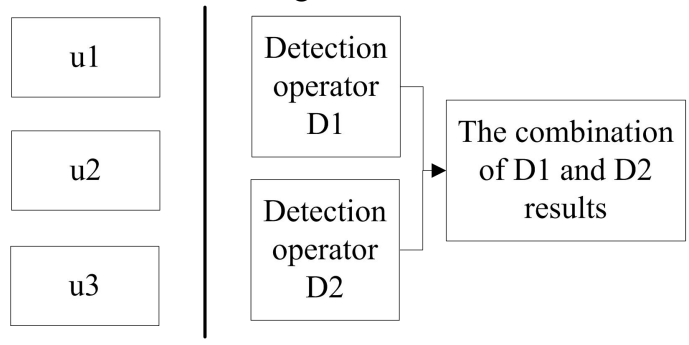

$\mathrm{u} 1, \mathrm{u} 2$ and $\mathrm{u} 3$ are the pixels

Figure 4. Edge detection and threshold segmentation process chart.

D1 operator is

$$
\begin{gathered}
r=\min \left(r_{12}, r_{13}\right) \\
r_{i, j}=1-\min \left[\frac{u_{i}}{u_{j}}, \frac{u_{j}}{u_{i}}\right]
\end{gathered}
$$

Among them, $u_{i}$ represents the gray value of the $i$-th template region.

D2 operator is

$$
\begin{gathered}
\rho_{i, j}^{2}=\frac{\sigma_{x}^{2}}{\sigma_{A}^{2}} \\
\sigma_{\mathrm{x}}^{2}=\frac{n_{i} n_{j}}{n^{2}}\left(u_{i}-u_{j}\right)^{2} \\
\rho=\min \left(\rho_{12}, \rho_{23}\right) \\
\sigma_{A}^{2}=\frac{1}{n_{2}}\left[n_{i} n_{j}\left(u_{i}-u_{j}\right)^{2}+n\left(n_{i} \sigma_{i}^{2}+n_{j} \sigma_{j}^{2}\right)\right]
\end{gathered}
$$

After comprehensive two operators, there are 


$$
\sigma(x, y)=\frac{x y}{1-x-y+x y}
$$

Among them, $\sigma(\mathrm{x}, \mathrm{y})$ is the $\mathrm{D} 1$ template response $\mathrm{r}$ and $\mathrm{D} 2$ template response $\rho$ combination results, in which the template can be adjusted according to the need. This paper uses $8 * 8$ resolution template, the use of suitable resolution window can reduce the amount of computer operation, so we can choose the appropriate block according to the size of the object and the gray change size of the internal background. Edge crack detection and threshold segmentation results are shown in Figure 5.

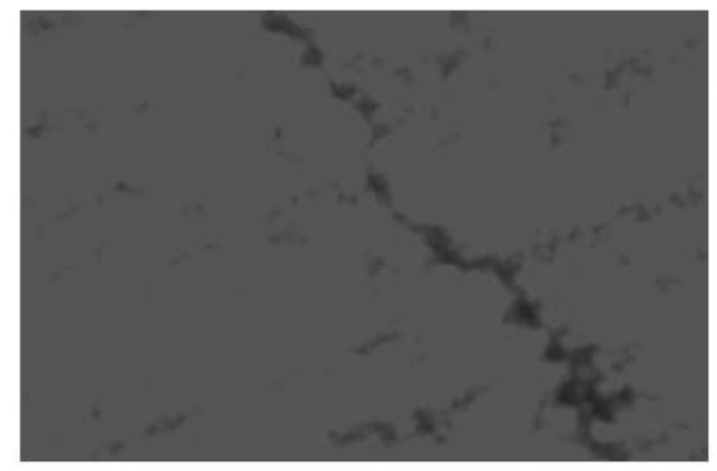

Figure 5. Effect of edge crack information detection and threshold segmentation.

Mechanical surface cracks generally need to detect the image, but some of the cracks are continuous, so we need to fill the process after the threshold segmentation, and the crack points are filled with repair, so that the image is a clear point, it can accurately determine the surface cracks of mechanical parts. The actual calculation of the algorithm generally uses recursion, but the computer system resources is limited, if the number of recursive layers is too large, the system will overflow; when processing a slightly larger number of images are easy to make mistakes, we will get incorrect measurement results. So we can change the recursive algorithm to the non-recursive algorithm, which can greatly reduce the computation and improve the real-time detection capability.

After several processing procedures, the effect of the image information is better, but the detection of the edge points is still not too continuous. In particular, there is little difference between the normal part of the object and the crack, it will not detect the edge, and it will cause the error. However, this paper not only needs to detect crack length information, but also needs to further crack connection and marking [12]. The segmented image carries out be numbered, followed by setting a threshold, and then we will relabeling after less than $\theta$ threshold crack is removed; if two or more crack distance are less than $\theta$ threshold, we will connect the two crack, eventually we can get the crack patterns. Therefore, the selection of threshold $\theta$ is an important symbol for controlling the crack selection, in practical application we only need to change the threshold $\theta$ to detect the crack. Figure 6 (a) is an image after the connection.

In order to get more accurate and clear graphics features, we also need to refine the label and connected image, so that the length of the crack can be measured and determined its general direction [13]. We use the fast fine algorithm of two value image. In the refinement process, the use of edge tracking technology carries out the operation of edge points, so that the speed of image refinement is greatly improved.

When tracking the edge, the point will delete the judgment for every trace a point, if it is the deleted points, we will delete; the last tracking point is back to the starting point, we consider that it complete the edge of the refinement. We assumed that around the corresponding number of tracking point $\mathrm{P}$ is $\mathrm{i}(\mathrm{i}=0,1,2,3,4,5 \ldots)$, according to the refinement basic principles and the edge tracking characteristics, the expressions for the rules are listed as follows: 


$$
\left\{\begin{array}{l}
S_{i}=n_{i-1}\left(n_{i}+n_{i+1}+n_{i-2}+n_{i-3}\right)\left(n_{i+1}+\bar{n}_{i+2}\right)\left(n_{i-2}+\bar{n}_{i-4}\right)(i=1,3,5,7) \\
S_{\mathrm{i}}=n_{i-2}\left(n_{i}+n_{i-1}+n_{i+4}+n_{i+5}\right)\left(\bar{n}_{i+1}+n\right) \quad(i=2,4,6)
\end{array}\right.
$$

In formula (8), $n_{i}$ is 0 or $1, \bar{n}_{i}=1-n_{i}$; if $S_{i}=0$, the $P$ point is reserved, or deleted. The image after thinning is shown in Figure 6 (b).

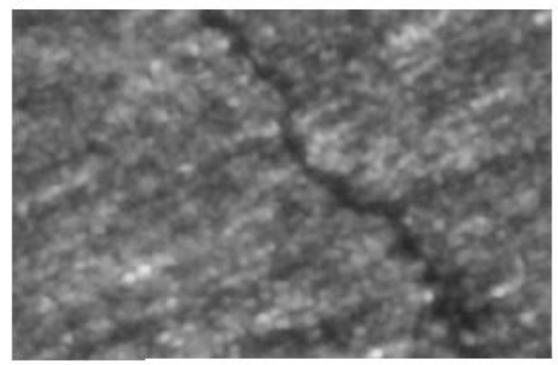

(a) Connection diagram

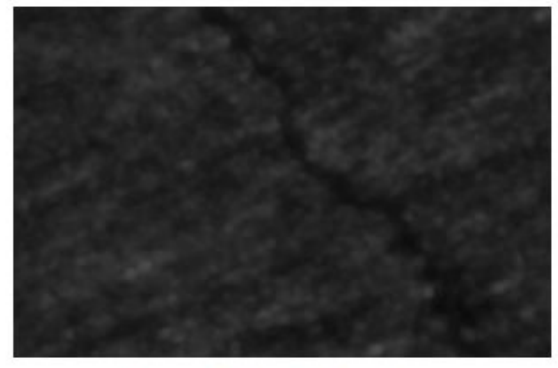

(b) Graphics after refinement

Figure 6. Graphical comparison after connection and refinement.

\section{Characteristic measurement}

Crack length calculation. By means of the above process, the crack region is separated, this paper can accurate to get the required crack area graphics, and the crack length can be obtained by traversing the method. In order to obtain the actual length of the crack, we first need to obtain the microscope magnification factor.

$$
L_{t}=L_{p} \times a
$$

Among them, $L t$ is the actual length of the crack; $L p$ is the length of the crack; a is the microscope magnification factor. Through the determination a value, detection system can calculate the actual length of the crack according to the algorithm factor.

Crack propagation rate calculation. In practical application, the fatigue crack is gradually expanded the surrounding from the surface, so the crack growth rate can be measured by the surface crack growth rate. By extracting the width of the crack trunk as a pixel, all the crack information in the two images is recorded for a certain time interval, and then this paper goes through the image information actual difference and time ratio to obtain the whole crack propagation rate

\section{Conclusions}

Through the mechanical part surface fatigue crack image, this paper carries out denoising, segmentation, patching, filtering, refinement and other processing experiments, the growth rate of crack length and surface fatigue crack are obtained by using the algorithm. Experiments also show that computer vision system is feasible in the application of surface crack detection, and it is worth applying to the reality. This paper designed a surface crack computer vision image detection method, its method not only can better detect the surface crack distribution, but also can accurately calculated crack length and expansion rate through this algorithm, this feature will be very important function on the practical application of the surface crack, which has great market prospects. 


\section{Acknowledgement}

It is a project supported by the Teaching research project of Hubei University of Police (JYKT2015014), the Science research project of Hubei University of Police (2014GZ005), the universities youth science and technology innovation team project of Hubei Province (T201421).

\section{References}

1. G.Y. Mo, D.W. Zuo, X.F. Li. Research on surface fatigue crack detection method based on CCD image. Mechanical manufacturing and automation, 2013(6): 55-57.

2. G.Y. Mo, D.W. Zuo, X.X. Zhu. Research on surface fatigue crack recognition and length calculation based on CCD image. Mechanical manufacturing and automation, 2014(2): 66-68.

3. X.J. Bi, S. Fan. Study of the image edge detection technology based on catastrophe theory. Application of Technology, 2013(6): 1-6.

4. Y.Q. Zou, G.C. Hou, F. Yang. Research on surface crack detection algorithm based on digital image processing. Micro computer information, 2014(4): 80-85.

5. B.B. Liu. Research on drosophila's compound eyes lesion image recognition system based on machine vision. Central South University, 2014: 1-13.

6. X.W. Kong. Application of the combined light source and image processing algorithm in the workpiece surface defect detection. Tianjin University, 2014: 1-9.

7. G.Y. Mo. Research on the application of CCD image recognition technology in fatigue crack detection. Nanjing University of Aeronautics \& Astronautics, 2014: 12-17.

8. X.Q. Chen, Y.H. Hu, Y.R. Huang. Image threshold segmentation based on two-dimensional maximum correlation criterion. Infrared and millimeter wave, 2014(5): 397-400.

9. X.Q. Chen, Y.H. Hu, Y.R. Huang. A two-dimensional maximum correlation criterion image threshold segmentation recursive algorithm. Computer engineering and applications, 2014(32): 95-97.

10. J.Y. Lin. Research on image segmentation algorithm based on Renyi entropy. Jilin University, 2014: 2-10.

11. S.Q. Zhang, Z.X. Meng, J.Z. Zhang. The extraction of micro fatigue crack image data based on edge detection. Journal of Northeast Forestry University, 2013(3): 111-112.

12. Y.M. Hu. Research on vehicle identification key technologies based on video. South China University of Technology, 2014: 2-9.

13. X.H. Zhu. Research on power equipment identification method based on image processing. North China Electric Power University (Hebei), 2014: 3-16. 